\title{
Surrounding motion affects the perceived locations of moving stimuli
}

\author{
David Whitney and Patrick Cavanagh \\ Vision Sciences Laboratory, Harvard University, Cambridge, MA, USA
}

\begin{abstract}
The perceived position of an object is determined not only by the retinal location of the object but also by gaze direction, eye movements, and the motion of the object itself. Recent evidence further suggests that the motion of one object can alter the perceived positions of stationary objects in remote regions of visual space (Whitney \& Cavanagh, 2000). This indicates that there is an influence of motion on perceived position, and that this influence can extend over large areas of the visual field. Yet, it remains unclear whether the motion of one object shifts the perceived positions of other moving stimuli. To test this we measured two well-known visual illusions, the Fröhlich effect and representational momentum, in the presence of extraneous surrounding motion. We found that the magnitude of these mislocalizations was altered depending on the direction and speed of the surrounding motion. The results indicate that the positions assigned to stationary and moving objects are affected by motion signals over large areas of space and that both types of stimuli may be assigned positions by a common mechanism.
\end{abstract}

There is growing evidence that the perceived position of an object is determined not only by the position and movements of the eyes (Cai, Pouget, Schlag-Rey, \& Schlag, 1997; Deubel, Schneider, \& Bridgeman, 1996; Matin, 1972; Ross, Morrone, \& Burr, 1997) and the object's retinal location (Lotze, 1886), but also by the object's motion. For example, the initial position of a moving object can appear shifted in the direction of motion (Fröhlich, 1929; Müsseler, Stork, \& Kerzel, this issue); the position of a moving stimulus can appear to lead a stationary flashed target (Hazelhoff, 1923, 1924; MacKay, 1958; Metzger, 1932; Nijhawan, 1994; see also Matin, Boff, \& Pola, 1976); and a stationary patch filled with moving texture appears not only to move, but also

Please address all correspondenc e to D. Whitney, Department of Psychology, The University of Western Ontario, N6A 5C2, Canada. Email: dvw@uwo.ca

This work was supported by a National Defence Science and Engineering Graduate Fellowship to DW, and NIH grant EY02598 to PC. Parts of this work were presented at the RepMo conference, August 2000, organized by Ian Thornton and Tim Hubbard. 
to be positionally shifted in the direction of its motion (DeValois \& DeValois, 1991; Ramachandran \& Anstis, 1990). In all of these phenomena, the position of a moving object appears displaced. Yet, recent evidence suggests that these position shifts may not be specific to the moving stimuli themselves. In fact, the motion of one object can influence the perceived position of another object, even when this second object is stationary and located a substantial distance away from the motion (Whitney \& Cavanagh, 2000). Therefore, the visual system not only uses local motion information, but also accesses distant motion signals when determining relative positions.

Although the perceived motion of one object is determined by the motions of other proximal objects, (Duncker, 1929/1938; Nakayama \& Silverman, 1984; Reinhard-Rutland, 1988; Snowden \& Braddick, 1989a,b), only recently has a similar process been implicated in the assignment of an object's location (Whitney \& Cavanagh, 2000). If the assigned location of an object requires a reference to surrounding motion signals, then not only should stationary objects be shifted in position by extraneous motion, but the positions of moving objects should be shifted as well.

To test whether the position of a moving object is shifted by extraneous motion-motion that does not belong to the object itself-we employed variants of two well-known visual illusions: the Fröhlich effect and representational momentum. The Fröhlich effect, mentioned earlier, is the apparent displacement of the initial position of an object when it appears and begins to move (Fröhlich, 1929; see Figure 1A). Representational momentum originally referred to the mislocalization of an object in the direction of its implied motion after it disappears (Freyd, 1987; Freyd \& Finke, 1984; Hubbard \& Bharucha, 1988; Kelly \& Freyd, 1987; cf., Freyd, 1983a,b). Subsequent studies have suggested that the perceived disappearance of a continuously moving object also appears displaced in the direction of its motion (Hubbard \& Bharucha, 1988; Hubbard \& Ruppel, this issue; see Figure 1B), though recent work has questioned the cause of this phenomenon (Kerzel, 2000; Kerzel, Jordan, \& Müsseler, 2001; Kerzel, Müsseler, \& Stork, 2000). To determine the influence of extraneous motion on the perceived positions of moving stimuli, we measured the Fröhlich effect and in the presence of surrounding motion signals.

\section{EXPERIMENT 1: Influence of surrounding motion on the Fröhlich effect}

\section{Methods}

Two naïve subjects and one of the authors (DVW) participated in the experiment. All were practised psychophysical observers. Each had normal or corrected-to-normal vision. Subjects were seated in a darkened experimental 

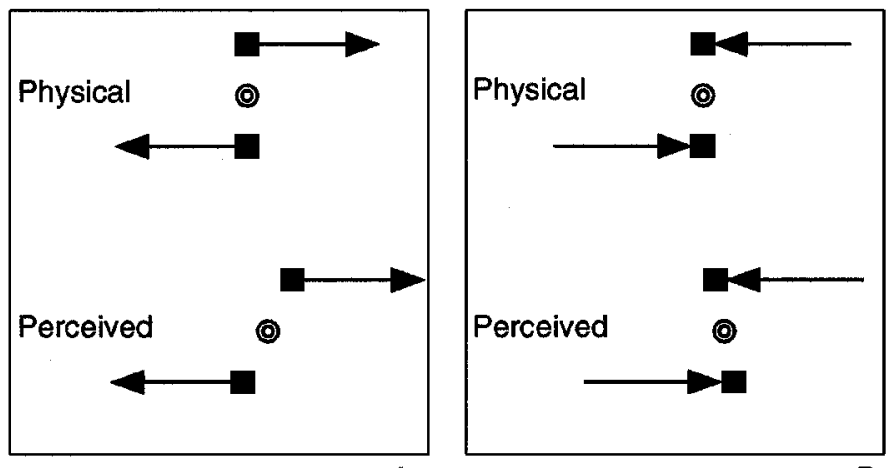

A

B

Figure 1. (A) A variation of the Fröhlich effect. Two bars (black squares) abruptly appear, moving in opposite directions. Though the bars are initially physically aligned, each appears displaced in the direction of its motion, creating a gap between them. The original Fröhlich effect was only measured with one moving bar, so the Fröhlich effect is doubled for this stimulus. The advantage of using two bars rather than one is that eye movements can be ruled out as a possible explanation for the effect. (B) The repesentational momentum of a continuously moving stimulus. In the traditional stimulus, a single moving bar abruptly disappears. Observers mislocalize the disappearanc e as being shifted in the direction of motion. Using two bars, that move toward each other and disappear, should double the mislocalization. Any effect that is found with this stimulus could not be due to eye movements, as the eye cannot simultaneously move or track targets in different directions.

booth with a chin rest $28 \mathrm{~cm}$ from a NEC Multisync P1150 CRT that had a refresh rate of $75 \mathrm{~Hz}$. A pair of white $\left(34.5 \mathrm{~cd} / \mathrm{m}^{2}\right)$ bars $\left(2.5^{\circ}\right.$ square $)$ appeared simultaneously and abruptly began to translate horizontally in opposite directions away from each other at 5.6,16.9, or $38.5 \mathrm{deg} / \mathrm{s}$ (Figure 1A; the direction was randomized across trials). The bars were vertically separated by $20^{\circ}$. A fixation point was provided at the centre of the display. A translating sine-wave grating (which we will call a linear grating, 0.14 cyc/deg) was presented above and below $\left( \pm 15.0^{\circ}\right.$ and $\pm 5.25^{\circ}$ from fixation) each of the bars, for a total of four gratings (each $60.0^{\circ} \times 6.0^{\circ}$ ). These two pairs of linear gratings (one above fixation and one below) always translated in opposite directions, and each pair of gratings could translate in the same or opposite direction as the nearest bar (Figure 2).

The initial horizontal position of each bar was varied from trial to trial and subjects were asked to judge whether the apparent initial positions of the bars overlapped (i.e., whether the bars ever appeared vertically aligned-2AFC task). At the beginning of each trial, the two pairs of linear gratings translated for $\sim 500 \mathrm{~ms}$ before the two bars were presented. The linear gratings could move at one of seven randomly determined speeds, either with or against the direction of the bars' motions (see Figure 2 for examples). There were six possible positions in which the bars could appear (six degrees of overlap, in multiples of $0.73^{\circ}$ ), and there were 30 trials for each of these positions. Psychometric 


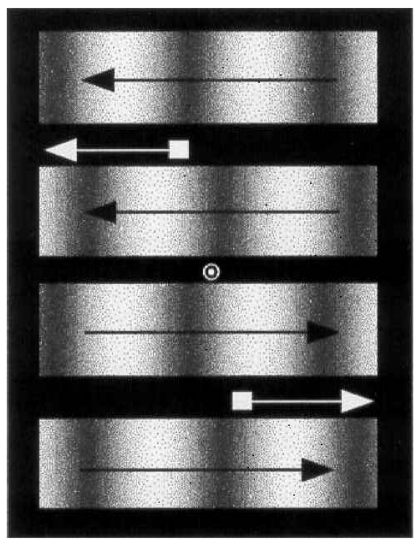

A

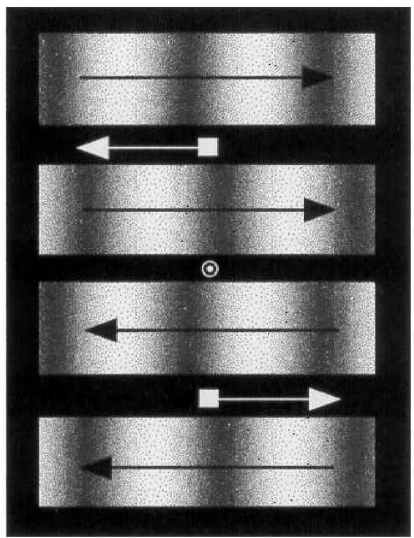

B

Figure 2. Stimulus used in the first experiment. A pair of translating linear gratings surrounded each of the bars (white squares). The gratings could move in a direction that was the same as, or opposite to, that of the bars. (A) When the gratings moved with the bars, the Fröhlich effect was exaggerated. (B) When the gratings moved opposite the bars, the Fröhlich effect was reduced or eliminated. The bars, in this image, were initially presented in physical alignment; they are perceived at different locations depending on the direction of the surrounding motion.

functions were fitted to the data, in each of the seven conditions, from the logistic function $y=\{1+\exp [-a(x-b)]\}^{-1}$, where $b$ estimates the degree of physical overlap between the bars in order for their initial positions to appear aligned.

\section{Results}

Figure 2 shows that the perceived Fröhlich gap between the bars depended on the direction of the surrounding gratings' motion. Though the bars were initially aligned, they appeared misaligned in a direction consistent with that of the surrounding gratings' motion.

Figure 3 shows the results of the first experiment. The abscissa on each graph shows the speed of the surrounding gratings, which were either in the direction of the bars' motions or opposite them ( 0 is a stationary grating; the open symbols represent the Fröhlich effect when no grating is present). The ordinate shows the Fröhlich effect in degrees. This was calculated as the gap between the two oppositely moving bars divided by two, since there were two moving stimuli rather than one. The three graphs show data for three different bar speeds.

The direction of the surrounding gratings' motion strongly influenced the perceived Fröhlich effect. When the gratings moved in a direction opposite that of the two bars, the Fröhlich effect was slightly reduced. Likewise, when the surrounding gratings moved in the direction of the bars' motion, the Fröhlich effect was amplified. The influence of the surrounding motion was noticeably 

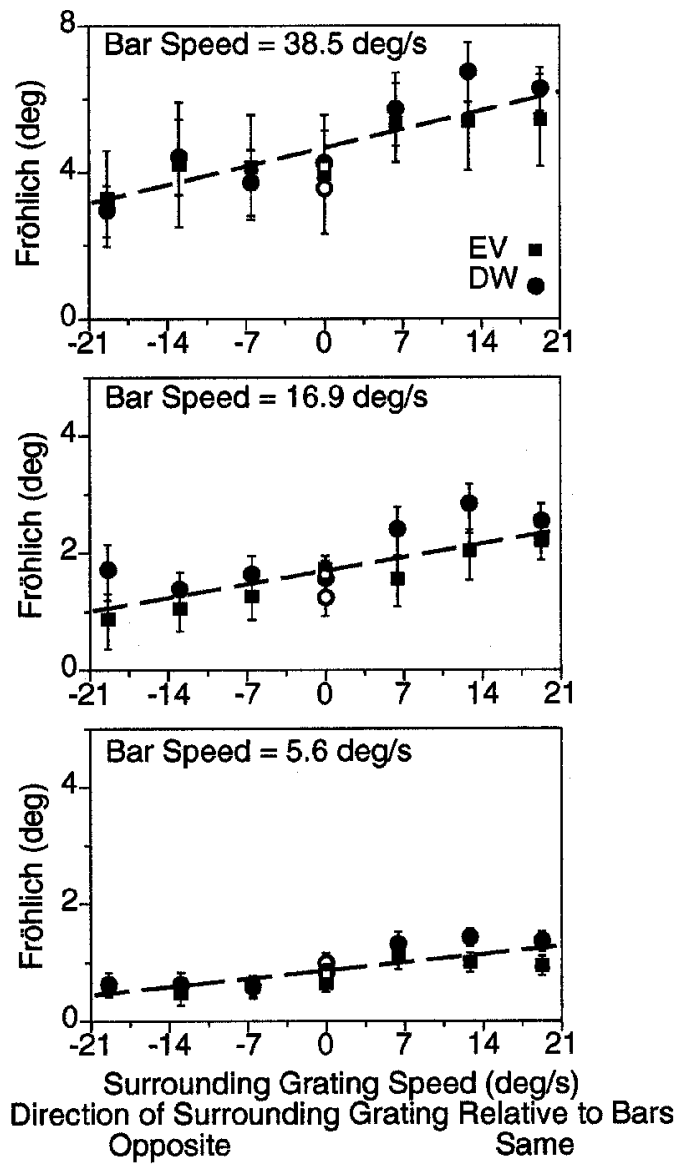

Figure 3. Results of the first experiment for two subjects. Data for a third subject were similar. The three graphs show results for three different bar speeds. The abscissa on each graph shows the speed and direction of the surrounding gratings' motion. The Fröhlich effect is plotted along the ordinate. The open circle and square in each graph show the Fröhlich effect when no surrounding gratings were presented. The filled circles and squares show the Fröhlich effect when the surrounding gratings were presented. Higher bar speeds resulted in a larger Fröhlich effect. The dashed line in each graph is a linear fit to the averaged data, and shows that there is a strong effect of the surrounding motion on the magnitude of the Fröhlich effect, particularly at high bar speeds. (Curve fits: bottom graph, $y=0.14 * x+0.86$; middle graph, $\mathrm{y}=0.23^{*} \mathrm{x}+1.70$; top graph, $\mathrm{y}=0.52 * \mathrm{x}+4.64$.) The error bars show $95 \%$ confidenc e intervals.

stronger when the bar speed was 38.5 or $16.9 \mathrm{deg} / \mathrm{s}$ (top two graphs in Figure $3)$. The dashed line in each graph of Figure 3 shows a linear fit to the averaged data, and the slope of the line provides an idea of the surrounding gratings' influence.

The results clearly showed that the spatially extraneous motion of the gratings influenced the localization of the moving bars. The influence of the 
gratings' motion was noticeably stronger when the bar and grating moved in the same direction. When the grating was stationary, the Fröhlich effect was very similar to that when no gratings were presented (compare open and filled symbols in Figure 3). The effect found here was not due to eye movements because the two bars moved in opposite directions, and the eye cannot move or rotate in two directions simultaneously.

\section{EXPERIMENT 2: \\ Influence of surrounding motion on representational momentum}

\section{Methods}

Subjects and experimental setup were identical to those in the first experiment. The stimulus was a slight variation: a pair of white $\left(16.2 \mathrm{~cd} / \mathrm{m}^{2}\right)$ bars $\left(2.5^{\circ}\right.$ square) translated horizontally in opposite directions toward each other at 5.6, 12.8 , or $38.5 \mathrm{deg} / \mathrm{s}$ (as in Figure 1B, the direction was randomized across trials; in the first experiment the bars moved away from each other). The bars translated toward each other until they were nearly vertically aligned, then abruptly disappeared. The bars were vertically separated by $20^{\circ}$. A fixation point was provided at the centre of the display. Just as in the first experiment, a translating linear grating was presented above and below each of the bars. These two pairs of linear gratings always translated in opposite directions (Figure 4), and each pair of gratings could translate in the same or opposite direction as the nearest bar.

In each trial, the two pairs of translating linear gratings continued to move for $\sim 650 \mathrm{~ms}$ after the bars disappeared. Subjects were asked to judge whether the endpoints of the bars' motions overlapped (i.e., whether the bars were recalled to be vertically aligned-2AFC task). Subjects were allowed to respond immediately after the bars disappeared. The interval between the bars' disappearances and the response was not systematically varied. In each trial, the two pairs of linear gratings could move at one of seven randomly determined speeds, either with or against the direction of the bars' motions. There were six possible positions in which the bars could disappear (six degrees of overlap), and there were 30 trials for each of these positions. Psychometric functions were fitted to the data, in each of the seven conditions, from the logistic function described above.

\section{Results}

Figure 5 shows that, similar to the first experiment, the surrounding gratings influenced the subjects' mislocalizations of the bars' disappearances. For example, when the bar moved at $38.5 \mathrm{deg} / \mathrm{s}$ (top graph in Figure 5), its final position was influenced by the direction of the surrounding gratings' motion. 


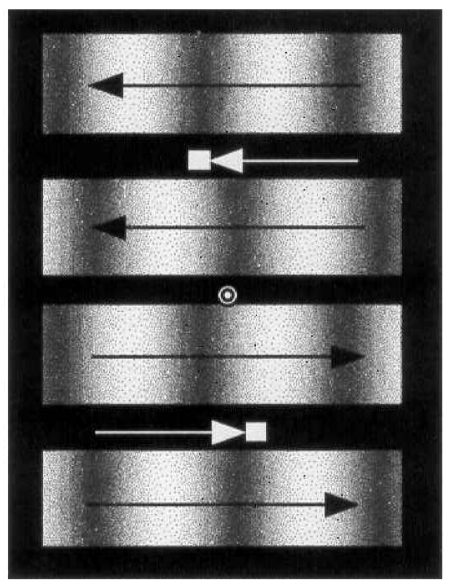

A

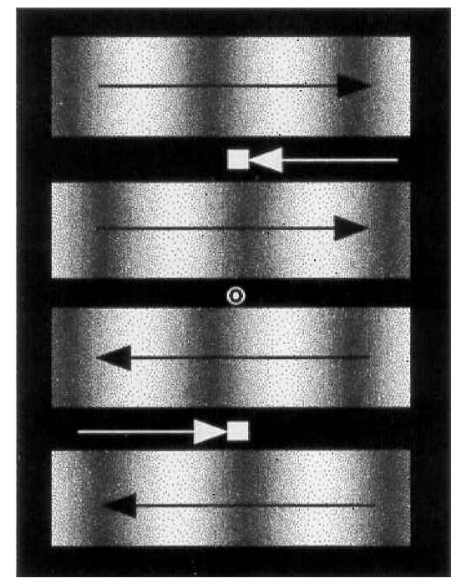

B

Figure 4. Stimulus used in the second experiment. As in the first experiment, a pair of translating linear gratings surrounde $d$ each of the bars (white squares). The gratings could move in a direction that was the same as, or opposite to, that of the bars. (A) When the gratings moved with the bars, the bars' final positions appeared displaced in the direction of motion. (B) When the gratings moved opposite the bars, the displacement was reduced or eliminated. The bars, in this image, had final positions that were physically aligned; they are perceived at different locations depending on the direction of the surrounding motion.

When the surrounding gratings moved in a direction consistent with that of the bars, the bars appeared to continue traveling beyond their actual disappearance point. The slope of the linear fit line through the averaged data (dashed line) gives an idea of the strength of the effect. The influence of the surrounding motion was weak for the slowest tested bar speed (bottom graph in Figure 5). Interestingly, the effect was stronger when the surrounding motion was in the same direction as the bar. As in the first experiment, since the two bars moved in opposite directions, the phenomenon reported here must not be due to eye movements.

Recent studies have shown that when eye movements are controlled for, there is very little, if any, illusory displacement of an object in the direction of its motion after it disappears (Kerzel, 2000; Kerzel et al., 2000, 2001). The results of the second experiment were consistent with this finding. For example, when the surrounding gratings were either stationary or not present (Figure 5 , grating speed $=0$ and open symbols, respectively), the displacement was virtually non-existent, except at speeds around $40 \mathrm{deg} / \mathrm{s}$, and even this was not significant (the most significant effect was for subject DVW, open circle in top graph of Figure 5: $t(1)=2.75, p>.05$ ). Eye movements therefore appear to be important in the localization of a moving object's final position (Mitrani, Dimitrov, Yakimoff, \& Mateeff, 1979). An additional factor that could contribute to the lack of displacement, in both the current study and those of Kerzel 

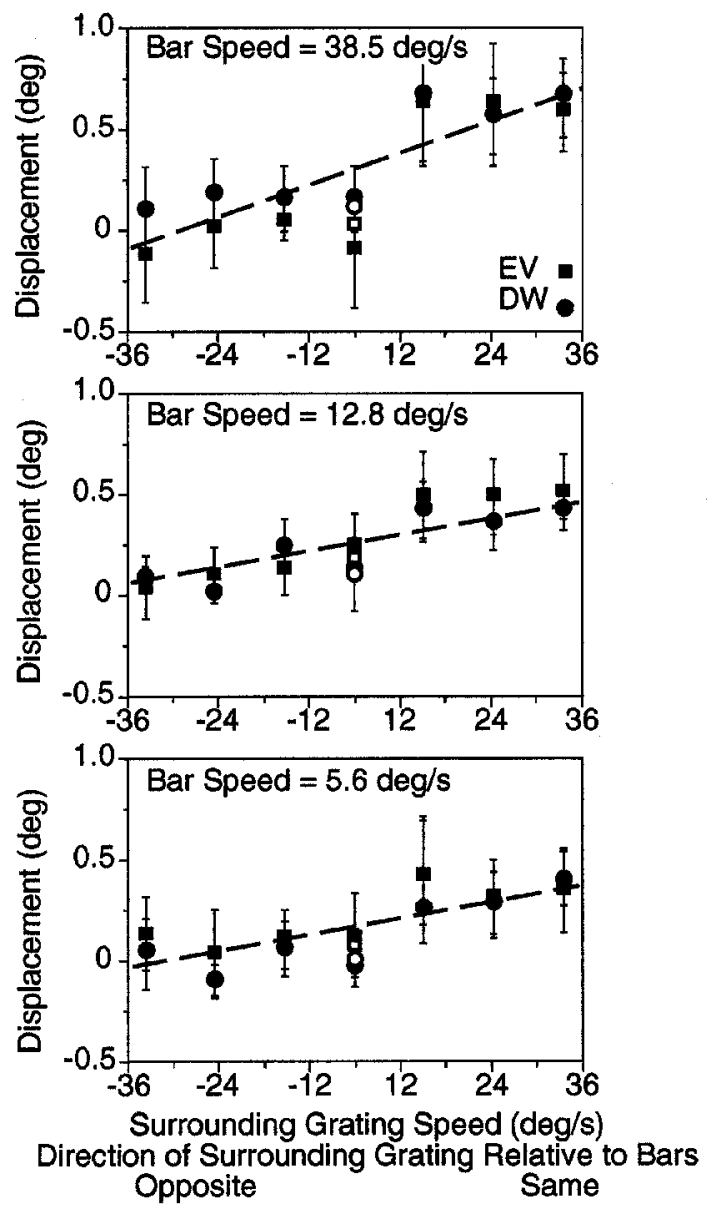

Figure 5. Results of the second experiment for two subjects. Data for a third subject were similar. The three graphs show results for three different bar speeds. The abscissa on each graph shows the speed and direction of the surrounding gratings' motion. The mislocalization of the bars' final positions (displacement) is plotted along the ordinate. Open and filled symbols refer to conditions where the surrounding grating was absent or present, respectively. Higher bar speeds had a negligible effect on the perceived mislocalization (when the grating is stationary or absent there is very little forward displacement, and this changes very little when bar speeds are increased). The dashed line in each graph is a linear fit to the averaged data, and shows that, at high bar speeds, there is an effect of the surrounding motion on the magnitude of the displacement effect. (Curve fits: bottom graph, $y=0.04 * x+0.17$; middle graph, $y=$ $0.05 * x+0.27$; top graph, $y=0.08 * x+0.29$.) The error bars show $95 \%$ confidence intervals. 
and colleagues, is the presence of a fixation point. The fixation point could inadvertently change the task, possibly by allowing subjects to judge the relative rather than the absolute location of the moving object. Regardless of what causes the reduced displacement reported here, it is consistent with previous studies that used the flash-lag effect to measure the perceived disappearance of a moving stimulus (Eagleman \& Sejnowski, 2000; Whitney, Murakami, \& Cavanagh, 2000). Thus, a variety of controlled stimuli show that, when fixating, the position of an object that abruptly stops or disappears is accurately perceived.

In the present study, however, we were not interested in the existence of representational momentum or displacements per se, but, rather, whether the motion signals of one object interact with the apparent position of another moving object. The results clearly show that, although no traditional displacement occurred, there was a strong effect of the surrounding motion on the positions of the bars' disappearances, as long as the bars moved at high speeds. This raises the interesting question of how, if at all, the two effects may be related. We will address this issue in the next section.

\section{DISCUSSION}

The results demonstrated that surrounding extraneous motion signals can influence the perceived positions of moving objects; that is, the position of a moving object's appearance or disappearance was shifted in the direction of the nearest or predominant direction of motion. This extends previous literature by showing that the positions of moving objects are not determined solely by their position and motion, but also by the motion of other objects in the field.

The results are in some ways consistent with many well-known phenomena. For example, induced motion (Duncker, 1929/1938; Reinhardt-Rutland, 1988) and motion capture (Ramachandran, 1987; Ramachandran \& Inada, 1985), where the motion of one object influences the perceived speed of another object, show that the influence of motion extends over large areas of space. (Induced motion is in a direction opposite that of the mislocalization we report, but may lie on a continuum with motion capture, cf., Murakami \& Shimojo, 1993). The motion of an object can also have a more spatially localized effect on the perceived speed of other objects. For example, motion integration and recruitment mechanisms (both spatial and temporal) are thought to explain the improvement in detection and discrimination of moving stimuli (Festa \& Welch, 1997; Lappin \& Bell, 1976; Nakayama \& Silverman, 1984; Sekuler, Sekuler, \& Sekuler, 1990; Snowden \& Braddick, 1989a,b; Watamaniuk, Sekuler \& Williams, 1989).

Yet, the present experiments suggest that perceived position, not just speed, is influenced by motion signals from extended regions of visual space. One might argue that the surrounding gratings "capture" the apparent speed of the 
moving bars and this causes the shift in perceived position (Ramachandran \& Anstis, 1990). In the second experiment, however, altering the physical speed of the bars did not change the mislocalization when there was no surrounding motion (open symbols in Figure 5). Thus, even if the surrounding gratings had captured the apparent speed of the bars, this should not have affected the mislocalization. Contrary to the motion capture argument, the surrounding gratings strongly influenced the bars' final positions (solid symbols in Figure 5). This is consistent with previous work showing that, even without motion capture, stationary objects can be shifted in position by extraneous motion (Whitney \& Cavanagh, 2000). Since the positions of both moving and stationary stimuli are influenced by motion signals, it is possible that relative position assignment for moving and stationary stimuli is accomplished by a single mechanism. Future studies are required to address this issue directly.

A remaining question is whether the moving gratings could have created a frame of reference, and whether this might have influenced the results. For example, when an object is briefly presented before and after a surrounding frame is displaced in position, the apparent location of the object appears to be shifted in a direction opposite that of the frame (Wong \& Mack, 1981; cf., Duncker, 1929/1938). Previous studies have also shown that a surrounding frame of reference that implies motion but does not physically move can influence the degree of representational momentum (Hubbard, 1993). If there were a frame of reference shift generated by the moving gratings, and the final position of the moving bar were judged relative to this frame, we might expect the apparent relative position of the bar to vary as a function of the surrounding motion. However, in the experiments reported here, there were two sets of gratings that moved in opposite directions, which rules out any single frame of reference. Also, the moving bars did not appear to travel along oblique trajectories, as a rotated frame of reference would predict (Hughes, Brecher, \& Fishkin, 1972). The stimulus used in the present study also sets any two hypothetical frames of reference in opposition (one frame shifts in one direction while the other shifts in the opposite direction), which reduces the salience of the effect (Rock, 1990). Further, there were other frames of reference visible, including the monitor, which is known to reduce or abolish the influence of a shifted frame (Rock, 1990). It is therefore unlikely that a frame of reference could explain the results reported here.

\section{The influence of motion on position, and its relation to representational momentum}

Though representational momentum with continuously moving stimuli has been replicated a number of times, recent work has shown that when eye movements are measured and eliminated, there is virtually no mislocalization 
(Kerzel, 2000; Kerzel, et al., 2000, 2001). The present results are consistent with this conclusion, as our second experiment found no traditional momentum effect when eye movements were controlled. Of course, this does not mean that there is no representational momentum (in the sense of Freyd \& Finke, 1984), it simply means that continuous motion may not be sufficient to elicit the phenomenon.

In fact, if eye movements contribute to, or cause, the mislocalization of a moving object's final position, this would be interesting for other reasons. Rather than revealing something about dynamic representations themselves (Freyd, 1987) or how the visual system internalizes physical principles (Hubbard, 1990; 1995a,b), the displacement may be a product of the interaction between the representations of moving objects and the predictive eye movements involved in tracking these objects. The visual system must, in many circumstances, make predictive judgements about where an object will be in order to successfully track it (Pavel, 1990; Skavenski, 1990), and there is accumulating evidence that both attentional shifts (Kowler, 1990; Kustov \& Robinson, 1996) and receptive field modulations and shifts (Duhamel, Colby, \& Goldberg, 1992) occur prior to eye movements. If there were a predictive process during smooth pursuit, then it might make sense for the eye to overshoot the disappearance of a moving stimulus (precisely what was found by Mitrani et al., 1979). This would, in turn, cause the apparent momentum of a continuously moving object.

Though this type of predictive eye movement account is able to explain the mislocalization of a moving object's final position, it is unable to explain the phenomenon that we report - that the position of one moving object can appear shifted by the motion of another object. The second experiment showed that surrounding motion influenced the localization of the moving bars' final positions, even though eye movements were controlled and could not have contributed to the results. Therefore, the phenomenon that we report appears to be due to a mechanism distinct from that which causes the representational momentum of continuously moving objects, although future work will resolve this conclusively.

\section{CONCLUSION}

The experiments reported here show that the motion of one object can influence the perceived positions of other moving stimuli. Assigning relative positions to moving objects therefore involves accessing predominant motion signals throughout large regions of the visual field. Along with previous results, the present study suggests that relative positions may be assigned to both stationary and moving stimuli by a common mechanism. 


\section{REFERENCES}

Cai, R.H., Pouget, A., Schlag-Rey, M., \& Schlag, J. (1997). Perceived geometrical relationship s affected by eye-movement signals. Nature, 386, 601-604.

Deubel, H., Schneider, W.X., \& Bridgeman, B. (1996). Postsaccadic target blanking prevents saccadic suppression of image displacement. Vision Research, 36, 985-996.

DeValois, R.L., \& DeValois, K.K. (1991). Vernier acuity with stationary moving gabors. Vision Research, 31, 1619-1626.

Duhamel, J.R., Colby, C.L., \& Goldberg, M.E. (1992). The updating of the representation of visual space in parietal cortex by intended eye movements. Science, 255, 90-92.

Duncker, K. (1938). Uber induzierte Bewegung (Ein Beitrag zur Theorie optisch wahrgenommener Bewegung). In W.D. Ellis (Ed. and Trans.), Source book of Gestalt psychology (pp. 161-172). London: Routledge \& Kegan Paul. (Original work published 1929.)

Eagleman, D.M., \& Sejnowski, T.J. (2000). Motion integration and postdiction in visual awareness. Science, 287, 2036-2038.

Festa, E.K., \& Welch, L. (1997). Recruitment mechanisms in speed and fine-directio n discrimination tasks. Vision Research, 37, 3129-3143.

Freyd, J.J. (1983a). The mental representation of movement when static stimuli are viewed. Perception and Psychophysics, 33, 575-581.

Freyd, J.J. (1983b). Representing the dynamic s of a static form. Memory and Cognition, 11, 342346.

Freyd, J.J. (1987). Dynamic mental representations . Psychological Review, 94, 427-438.

Freyd, J.J., \& Finke, R.A. (1984). Representational momentum. Journal of Experimental Psychology: Learning, Memory, and Cognition, 10, 126-132.

Fröhlich, F.W. (1929). Die Empfindungszeit. Jena, Germany: Verlag von Gustav Fischer.

Hazelhoff, F.F. (1923). De waarnemingstijd. Unpublished thesis, Groningen, The Netherlands.

Hazelhoff, F.F. (1924). Die Wahrnehmungszeit-I. Zeitschrift für die Psychologie und Physiologie der Sinnesorgane, 97, 174-190.

Hubbard, T.L. (1990). Cognitive representation of linear motion: Possible direction and gravity effects in judged displacement. Memory and Cognition, 18, 299-309.

Hubbard, T.L. (1993). The effect of context on visual representational momentum. Memory and Cognition, 21, 103-114.

Hubbard, T.L. (1995a). Cognitive representation of motion: Evidence for friction and gravity analogues. Journal of Experimental Psychology: Learning, Memory, and Cognition, 21, $241-254$.

Hubbard, T.L. (1995b). Environmental invariants in the representation of motion: Implied dynamics and representational momentum, gravity, friction, and centripetal force. Psychonomic Bulletin and Review, 2, 322-338.

Hubbard, T.L., \& Bharucha, J.J. (1988). Judged displacement in apparent vertical and horizontal motion. Perception and Psychophysic s, 44, 211-221.

Hubbard, T.L., \& Ruppel, S.E. (this issue). A possible role of naïve impetus in Michotte's "launching effect": Evidence from representational momentum. Visual Cognition, 9, 153176.

Hughes, P.C., Brecher, G.A., \& Fishkin, S.M. (1972). Effects of rotating background upon the perception of verticality. Perception and Psychophysic s, 11, 135-138.

Kelly, M.H., \& Freyd, J.J. (1987). Exploration s of representationa 1 momentum. Cognitive Psychology, 19, 369-401.

Kerzel, D. (2000). Eye movements and visible persistence explain the mislocalization of the final position of a moving target. Vision Research, 40, 3703-15.

Kerzel, D., Jordan, J.S., \& Müsseler, J. (2001). The role of perception in the mislocalization of the final position of a moving target. Journal of Experimental Psychology: Human Perception and Performance, 27, 829-840. 
Kerzel, D., Müsseler, J., \& Stork, S. (2000). The mislocationalization of the final position of a moving target: An error in memory and/or perception? RepMo, 14, (Suppl.).

Kowler, E. (1990). The role of visual and cognitive processes in the control of eye movement. In E. Kowler (Ed.), Eye movements and their role in visual and cognitive processes (pp. 1-70). Amsterdam: Elsevier.

Kustov, A.A., \& Robinson, D.L. (1996). Shared neural control of attentional shifts and eye movements. Nature, 384, 74-77.

Lappin, J.S., \& Bell, H.H. (1976). The detection of coherence in moving random-dot patterns. Vision Research, 16, 161-168.

Lotze, H. (1886). Outlines of psychology (G.T. Ladd, Trans.). Boston: Ginn.

MacKay, D.M. (1958). Perceptual stability of a stroboscopicall y lit visual field containing selfluminous objects. Nature, 181, 507-508.

Matin, L. (1972). Eye movements and perceived visual direction. In D. Jameson \& L. Hurvich (Eds.), Handbook of sensory physiology, Vol. VII/4 (pp. 331-380). Berlin: Springer-Verlag .

Matin, L., Boff, K., \& Pola, J. (1976). Vernier offset produce d by rotary target motion. Perception Psychophysics, 20, 138-142.

Metzger, W. (1932). Versuch einer gemeinsamen Theorie der Phänomene Fröhlichs und Hazelhoffs und Kritik ihrer Verfahren zur Messung der Empfindungszeit. Psychologische Forschung, 16, 176-200.

Mitrani, L., Dimitrov, G., Yakimoff, N., \& Mateeff, S. (1979). Oculomotor and perceptual localization during smooth eye movements. Vision Research, 19, 609-612.

Murakami, I., \& Shimojo, S. (1993). Motion capture changes to induced motion at higher luminance contrasts, smaller eccentricities, and larger inducer sizes. Vision Research, 33, 20912107.

Müsseler, J., Stork, S., \& Kerzel, D. (this issue). Comparing mislocalization s with moving stimuli: The Fröhlich effect, the flash-lag, and representationa 1 momentum. Visual Cognition, 9, $120-138$.

Nakayama, K., \& Silverman, G. (1984). Temporal and spatial characteristic s of the upper displacement limit for motion in random dots. Vision Research, 24, 293-300.

Nijhawan, R. (1994). Motion extrapolation in catching. Nature, 370, 256-257.

Pavel, M. (1990). Predictive control of eye movement. In E. Kowler (Ed.), Eye movements and their role in visual and cognitive processes (pp. 71-114). Amsterdam: Elsevier.

Ramachandran, V.S. (1987). Interaction between colour and motion in human vision. Nature, $328,645-647$.

Ramachandran, V.S., \& Anstis, S.M. (1990). Illusory displacement of equiluminou s kinetic edges. Perception, 19, 611-616.

Ramachandran, V.S., \& Inada, V. (1985). Spatial phase and frequency in motion capture of random-dot patterns. Spatial Vision, 1, 57-67.

Reinhardt-Rutland, A.H. (1988). Induced movement in the visual modality: An overview. Psychological Bulletin, 103, 57-71.

Rock, I. (1990). The frame of reference. In I. Rock (Ed.), The legacy of Solomon Asch (pp. 243268). Hillsdale, NJ: Lawrence Erlbaum Associates Inc.

Ross, J., Morrone, C., \& Burr, D.C. (1997). Compression of visual space before saccades. Nature, 386, 598-601.

Sekuler, A.B., Sekuler, R., \& Sekuler, E.B. (1990). How the visual system detects changes in the direction of moving targets. Perception, 19, 181-195.

Skavenski, A.A. (1990). Eye movement and visual localization of objects in space. In E. Kowler (Ed.), Eye movements and their role in visual and cognitive processes (pp. 263-287). Amsterdam: Elsevier.

Snowden, R.J., \& Braddick, O.J. (1989a). The combination of motion signals over time. Vision Research, 29, 1621-1630. 
Snowden, R.J., \& Braddick, O.J. (1989b). Extension of displacement limits in multiple-exposur e sequences of apparent motion. Vision Research, 29, 1777-1787.

Watamaniuk, S.N., Sekuler, R., \& Williams, D.W. (1989). Direction perception in complex dynamic displays: the integration of direction information. Vision Research, 29, 47-59.

Whitney, D., \& Cavanagh, P. (2000). Motion distorts visual space: Shifting the perceived positions of remote stationary objects. Nature Neuroscience, 3, 954-959.

Whitney, D., Murakami, I., \& Cavanagh, P. (2000). Illusory spatial offset of a flash relative to a moving stimulus is caused by differential latencies for moving and flashed stimuli. Vision Research, 40, 137-149.

Wong, E., \& Mack, A. (1981). Saccadic programming and perceived location. Acta Psychologic a (Amsterdam), 48, 123-131. 